\title{
Les habitudes
}

\section{de succion non nutritive chez l'enfant}

\section{RÉSUMÉ}

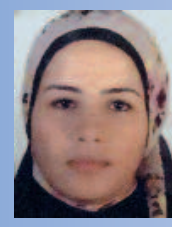

Afaf HOUB-DINE

Résidente en orthopédie dento-faciale, Faculté de médecine dentaire de Rabat, Université Mohamed V B.P 6212, Rabat-Instituts, Rabat, Maroc.

Loubna BAHIJE

Professeur assistant en orthopédie dento-faciale,

Faculté de médecine dentaire de Rabat, Université Mohamed V.

Mustapha EL ALLOUSSI

Professeur de l'enseignement supérieur en pédodontie-prévention,

Faculté de médecine dentaire de Rabat, Université Mohamed V.

\section{Fatima ZAOUI}

Professeur de l'enseignement supérieur et chef du service

d'orthopédie dento-faciale,

Faculté de médecine dentaire de Rabat, Université Mohamed V.

La succion chez l'enfant est décrite dans la littérature sous deux aspects : la succion nutritive et la succion non nutritive. La succion nutritive est relative à l'allaitement au sein, au biberon ou à une combinaison des deux. La succion non nutritive est évoquée lorsque l'enfant suce son pouce ou d'autres doigts, une tétine artificielle ou d'autres objets.

Les habitudes de succion non nutritive sont très fréquentes chez le jeune enfant. Bien qu'il ne faille pas dramatiser devant une succion digitale chez l'enfant de moins de 5 ans, celle-ci engendre toutefois des malocclusions à des degrés variables surtout avec l'éruption des dents définitives.

La succion non nutritive paraît donc complexe, tant dans ses conséquences que dans sa prise en charge. Plusieurs procédés et dispositifs utilisés pour l'arrêt de cette habitude déformante ont donné lieu à des discussions dans la littérature, les méthodes éducatives et psychologiques restent toujours d'actualité.

succion non nutritive

succion du pouce

succion de la tétine
AOS 2011;254:123-132

DOI: $10.1051 /$ aos/2011204

(C) AEOS / EDP Sciences 


\section{Introduction}

$>$

L'implication de la succion non nutritive

chez le jeune enfant comme facteur causal des malocclusions dentaires continue a être un sujet de controverse, de même que son étiologie. Il existe plusieurs théories :

- la théorie alimentaire qui voit une relation entre la forme de l'alimentation et la succion digitale ;

- la théorie psychanalytique : selon Freud, la succion procure un plaisir ;

- la théorie réflexe : pour laquelle il s'agit initialement d'un réflexe, tout contact avec la région buccale déclenchant la succion. Ce réflexe se transforme ensuite en une véritable praxie.

Si les effets de cette habitude de succion non nutritive durant la denture lactéale sont minimes, sa persistance jusqu'à l'apparition des dents permanentes peut être à l'origine de nombreuses anomalies dentaires et maxillofaciales. En effet, les os de la face sont d'origine conjonctive et leur développement est profondément influencé par l'environnement ainsi perturbé [1]. Avant le port de tout appareillage, le praticien doit parvenir à convaincre son jeune patient de la nécessité d'arrêter la succion.

\section{De la succion à la mastication}

La succion, phénomène réflexe, est la première fonction motrice à se mettre en place chez le fœtus humain [2]. Associée à la déglutition du liquide amniotique, elle permet l'élaboration du programme de succion-déglutition qui doit être opérationnel dès la naissance.

A la naissance, la succion est un indice qualitatif de maturation neurologique néonatale. Le lait (maternel ou aliment lacté premier âge) est l'aliment exclusif du nourrisson et la succion est la seule stratégie d'alimentation utilisable par le nouveau-né pendant les premiers mois de la vie. La diversification alimentaire fera évoluer cette fonction [3]. La succion assure donc l'oralité alimentaire jusqu'à l'apparition des premières dents, moment à partir duquel une nouvelle stratégie motrice doit se mettre en place.
La succion-déglutition est caractérisée par un contact entre la langue et les lèvres et ce contact restera physiologique tant que les incisives lactéales n'auront pas évolué. Mais à partir du moment où la denture lactéale se met en place, elle va délimiter deux territoires : celui de la langue et celui des lèvres, avec entre les deux une frontière infranchissable : les dents. Cependant, il arrive très souvent que malgré la présence de la barrière incisive, la langue continue à rechercher le contact labial et à perdurer la succion-déglutition. Le pouce n'est alors qu'un prétexte, un point d'appel à cette posture linguale dysfonctionnelle $[4,5]$.

Après 6 mois, avec l'apparition des dents et la diversification alimentaire, le besoin de succion ne représente plus un instinct mais un besoin de réconfort, un « consolateur ». 


\section{Facteurs de risque}

Séparé de ses parents, entouré par des étrangers ou dans un environnement peu familier, l'enfant trouve dans la succion du pouce ou de tout autre objet une forme de sécurité émotionnelle, elle peut également l'aider à dormir. C'est pour cette raison que l'on observe une fréquence de succion plus importante chez les enfants en bas âge le soir et quand ils sont fatigués [6].

Les résultats de l'étude menée par L. LupiPegurier et M. Muller-Bolla [1] confirment que les habitudes de succion pourraient être associées à la société moderne occidentale. L'exercice professionnel de la mère joue un rôle dans I'acquisition de cette mauvaise habitude $[1,7]$. Avec l'évolution du contexte socio-économique et les exigences du travail de la femme, la succion du pouce ou de la tétine est devenue assez fréquente. Les enfants paraissent très sensibles à leur environnement pendant la petite enfance [8].

La succion digitale semblerait liée au fait que les enfants soient toujours gardés par leur mère ou un autre membre de la famille. En effet, que ce soit à la crèche ou chez une gardienne, les enfants sont nombreux. Ils trouvent des compagnons de jeu. Ils sont plus actifs et n'éprouvent plus le besoin de s'isoler avec leur pouce. Le fait d'être rapidement en contact avec des enfants de leur âge développe leur sociabilité et limite le repli sur soi.

A l'inverse, lorsqu'ils sont gardés par un membre de leur famille, et a fortiori par leur mère, ils sont habitués à ce que l'on s'occupe davantage d'eux. Ceci créerait une sorte " d'accoutumance à l'affection ", et par là même, un besoin toujours plus grand de témoignages d'amour et de protection qui expliquerait le refuge dans la succion.

Par ailleurs, I'ambiance familiale est toujours plus sereine, moins bruyante que celle des garderies sans cesse animées par les cris et les rires des nombreux enfants. Ce contexte, rassurant et propice à l'assoupissement, serait favorable au développement d'une habitude de succion [1].

\section{Éléments de diagnostic}

L'enfant peut sucer son pouce ou un autre doigt, les joues (fig. 1), la langue, un linge ou un objet. L'interrogatoire des parents permet d'analyser certains facteurs qui auront une influence sur les effets physiologiques de la succion du pouce.

\section{La fréquence de la succion}

Le nombre de fois que l'enfant place son pouce dans sa bouche durant la journée.

\section{La durée}

de la succion

La date d'apparition de cette habitude, sa fréquence et son ancienneté (exemple : un enfant suce son pouce chaque soir avant de s'endormir pendant 20 à 25 minutes depuis qu'il avait six mois).

Il n'existe pas d'égalité devant la « dysmorphogénicité » d'une succion digitale : 2 minutes de 

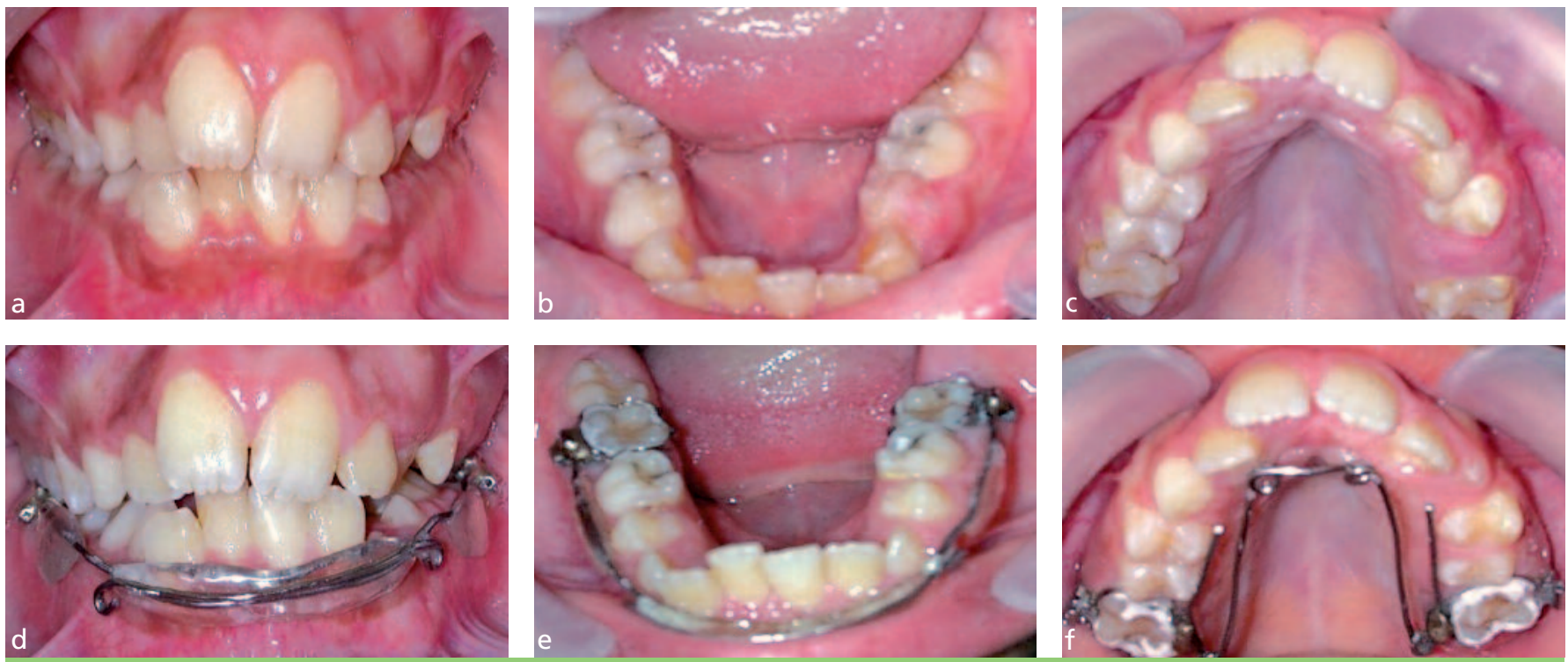

Fig. $1 \mathrm{a}$ à $\mathrm{f}$

a à C Patient âgé de 10 ans présentant une arcade mandibulaire en lyre (b) provoquée par la succion des joues.

d à f L'arrêt de cette habitude a été géré par motivation, explication et mise en place d'un lip bumper avec écrans latéraux à la mandibule $(d, e)$ et un quadhélix au maxillaire $(f)$.

succion par jour peuvent créer autant de dégâts qu'une succion quasi permanente. II faut noter, ici, la grande importance du terrain et donc de la donne génétique.

\section{Lintensité de la succion}

La force de la succion et la vigueur avec laquelle l'enfant suce son pouce.
La position du pouce

ou de l'objet sucé

L'effet de levier sera différent selon l'angulation du pouce et sa position dans la bouche. La poussée sur les structures anatomiques se fait à des endroits différents selon la longueur, la grosseur, la rotation, I'angulation et la flexibilité du pouce [9].

\section{Conséquences de la succion prolongée}

La succion persistante d'un doigt ou d'un objet au-delà d'un certain âge, outre qu'elle entraîne des déformations des arcades, peut avoir des conséquences sur les fonctions oro-faciales.

En effet, le passage de la succion-déglutition à une déglutition type sujet denté ne se réalise pas et les conséquences sont nombreuses (fonctionnelles, anatomiques). II existe alors une interposition linguale entre les arcades et les lèvres lors de certaines fonctions (succiondéglutition et phonation notamment) [10].

Bien qu'il ne faille pas dramatiser devant une succion digitale chez l'enfant de moins de 5 ans, celle-ci engendre toutefois des malocclusions à des degrés variables. Ces dernières sont interdépendantes de la position du doigt en bouche, 
des contractions musculaires associées, de la position mandibulaire pendant la succion, de la morphologie squelettique, de la durée et de la fréquence de la succion. Donc, il ne faut pas prendre cette habitude à la légère!

En effet, les déformations permanentes augmentent chez l'enfant qui suce son pouce après l'âge de trois ans. Il peut exister des anomalies dans les trois sens de l'espace : vertical, transversal et sagittal.

Les conséquences les plus fréquentes de cette habitude " néfaste " peuvent être regroupées en :

- anomalies occlusales : béance (fig. 2), proalvéolie supérieure (fig. 3), surplomb excessif, diastèmes incisifs [11] ; -déformations squelettiques : décalage squelettique de classe II, palais creux profond et étroit $[7,12]$;

- déformation digitale (fig. 4) ;

- problèmes fonctionnels : déglutition atypique systématique, ventilation buccale ;

- troubles lors de la prononciation de certains phonèmes : T-D-N-L-CH-S ;

- problèmes psychologiques (la peur d'être ridiculisé par les amis, le réflexe de se cacher pour sucer son pouce...).

La succion de la tétine (fig. 5) ou d'un linge est aussi néfaste, elle peut affecter les arcades dentaires de la même manière que la succion du pouce ou d'un autre doigt [7].
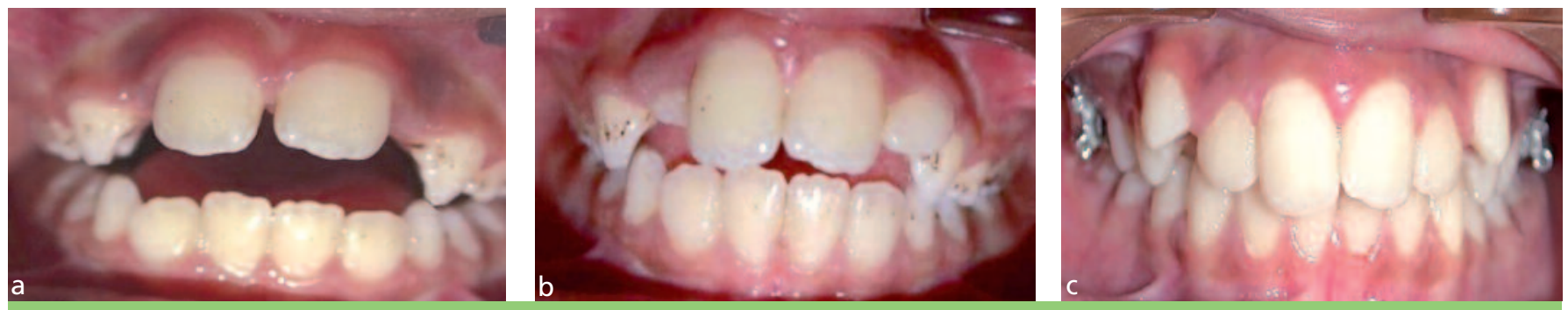

Fig. 2 a à c

a Patiente âgée de 9 ans présentant une béance antérieure provoquée par la succion du pouce.

b Résultat obtenu après 4 mois de motivation et de rééducation active.

Résultat stable après 3 ans.
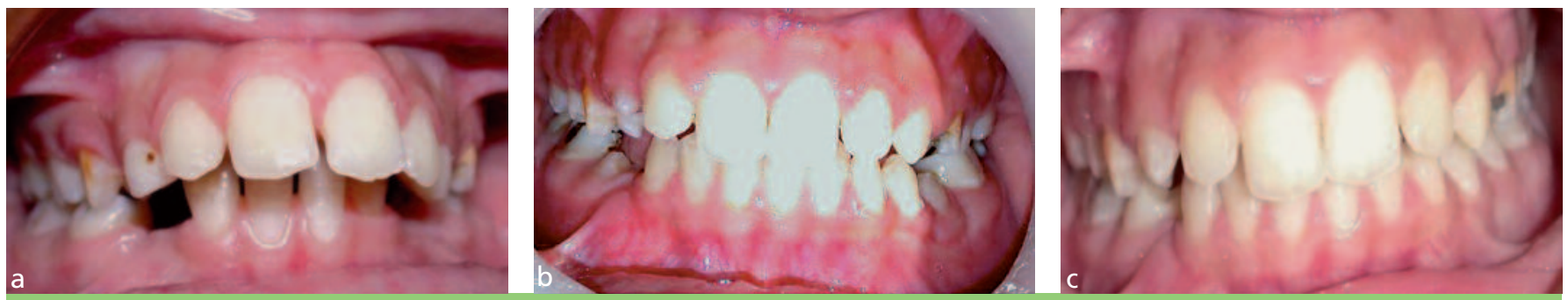

Fig. 3 a à c

a Proalvéolie supérieure provoquée par la succion du pouce chez une patiente âgée de 9 ans.

b Une diminution considérable de l'overjet après 9 mois de motivation et suivi.

c Stabilité du résultat après 37 mois. 


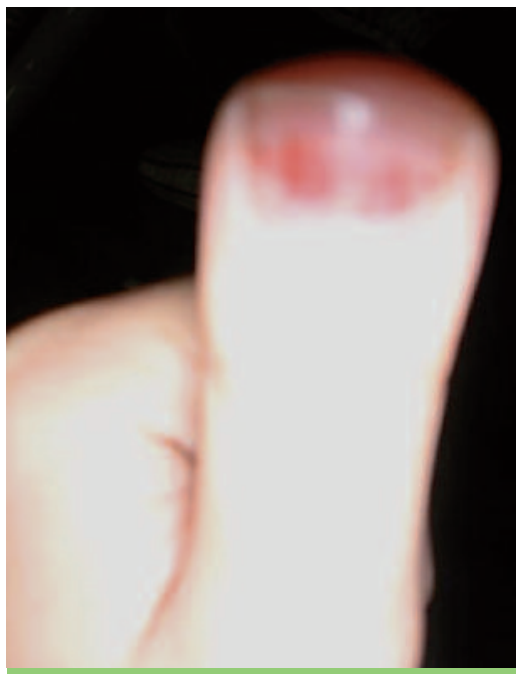

Fig. 4 Déformation du pouce sucé
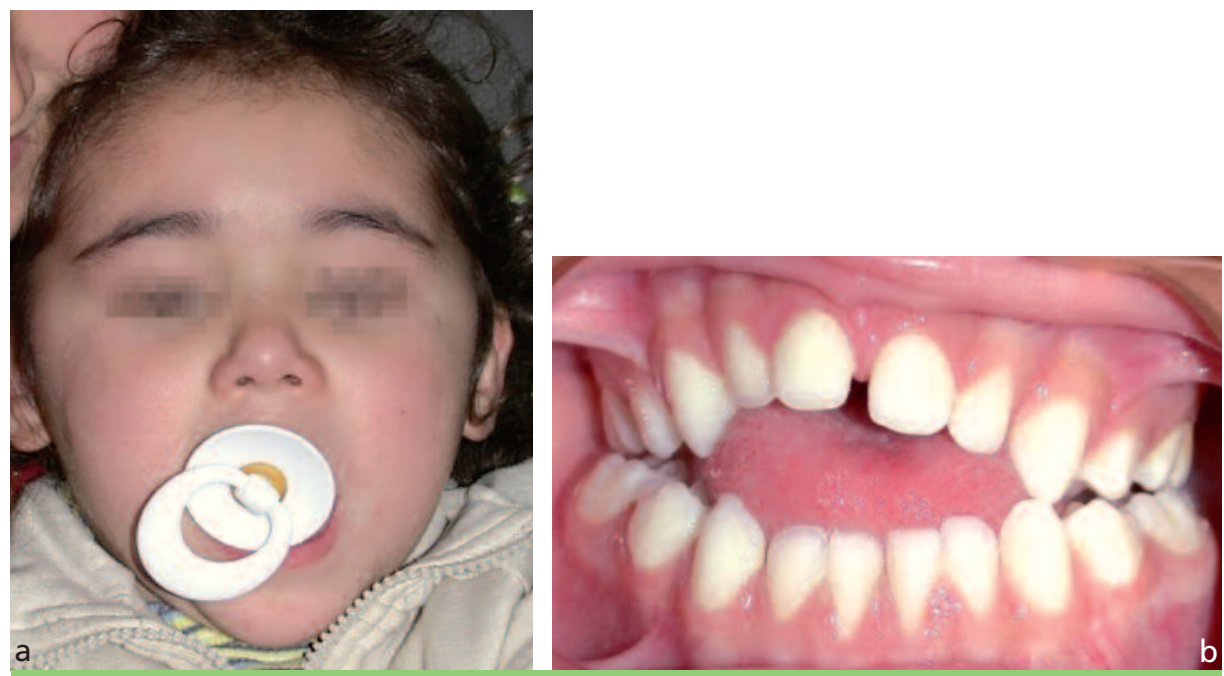

Fig. 5 a à $b$

a Jeune patiente de 4 ans et demi suce une tétine.

b On remarque la même dysmorphose provoquée par la succion digitale.
Cependant, la tétine a quelques avantages par rapport au pouce car elle peut présenter des formes évolutives avec l'âge de l'enfant et elle s'adapte à son palais. La tétine est par ailleurs plus hygiénique pour le jeune enfant qui commence à se déplacer, à toucher à tout, elle peut et doit en effet être stérilisée régulièrement.
Autre avantage de la tétine, son usage peut être contrôlé et régulé par les parents en limitant son utilisation à la sieste et au coucher par exemple. Cette limitation permet ensuite peu à peu d'aller vers l'abandon total de la sucette [7].

La succion du linge peut exposer l'enfant à une intoxication à partir des produits de lessive [13].

\section{Conséquences de la succion prolongée}

La succion prolongée du pouce inquiète les parents dont beaucoup se demandent comment parvenir à convaincre l'enfant d'arrêter. Tout d'abord, il faut considérer l'âge de l'enfant. Sucer quelque chose entre 2 et 8 ans, ne relève pas de la même problématique [10].

\section{Avant 4 ans}

Les chercheurs sont unanimes « Ne pas intervenir directement chez le jeune enfant avant 3 ans » car l'enfant serait privé d'une ressource importante et il serait obligé d'en trouver une autre. II faut donc souvent attendre qu'il ait atteint une maturité suffisante pour le convaincre de renoncer à cette habitude. La plupart des enfants cessent d'eux-mêmes l'habitude de succion entre l'âge de 2 et 4 ans, car ils passent plusieurs heures de réveil à explorer leur environnement. Si l'enfant ne s'arrête pas tout seul, les parents devraient l'aider à se débarrasser de cette habitude après l'âge de 4 ans. Le renforcement négatif (réprimande, punition) est généralement sans effet, rendant 
I'enfant défensif, et le ramenant à cette habitude [14].

À partir de 5 à 6 ans

Devant une attitude très autoritaire, l'enfant pourrait être porté à réagir en suçant son pouce. En effet, l'enfant qui a I'habitude de sucer son pouce le fait le plus souvent lorsqu'il est tendu. D'autre part, ce ne sont ni l'attention des parents ni le renforcement qui font que l'enfant suce son pouce, mais bien la satisfaction qu'il en éprouve. Ce n'est donc pas en ignorant le comportement de l'enfant qu'on parviendra à le faire cesser.

Les interventions utilisant le renforcement positif et la sensibilisation de l'enfant sont très efficaces. Il faut simplement aider l'enfant à abandonner le pouce ou le doigt par des encouragements de la part, et de son entourage familial, et du praticien (prendre le temps de converser avec lui) et surtout éviter de le culpabiliser. On demande à l'enfant d'abandonner une habitude qu'il affectionnait et qui le sécu- risait, il faut donc lui demander de cesser de le faire à des périodes où il n'est pas fatigué ou frustré durant le jour, miser sur les récompenses et l'encouragement, mettre en place un programme interactif, valoriser et soutenir l'enfant, et bannir les punitions.

Le jeu permet une relation parent-enfant saine pour remédier à cette habitude, l'enfant peut dessiner sur le doigt concerné (avec la participation des parents et du frère ou de la sœur), le visage d'un personnage qui l'aidera à ne pas mettre son doigt à la bouche (fig. 6). Cette attitude permet une évolution favorable vers l'arrêt de ce tic, et aide le petit patient dans sa maturation psychoaffective [14].

L'enfant qui aime ce qu'il fait et qui est absorbé par des activités exigeant l'emploi de ses deux mains se trouve forcément dans l'impossibilité de sucer son pouce. Prévoyez donc le genre d'activité pour laquelle l'enfant doit se servir de ses deux mains.

La méthode du Calendrier ou Carnet (fig. 7) inspirée des thérapies comportementales pour

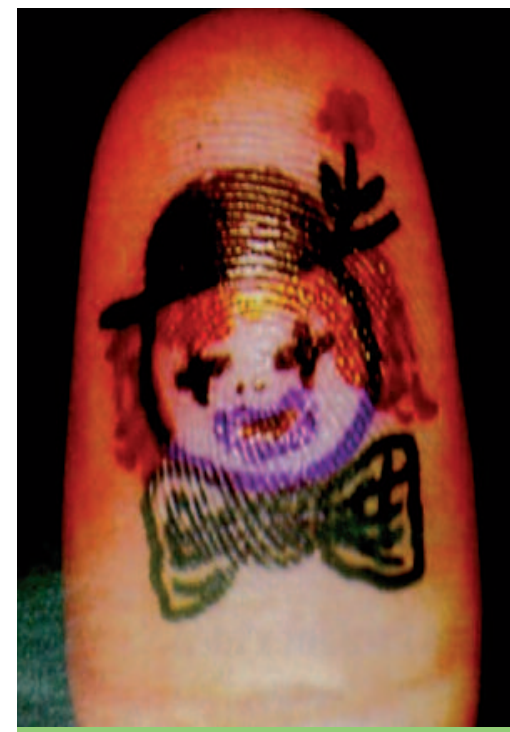

Fig. 6 dessin sur le pouce [15].

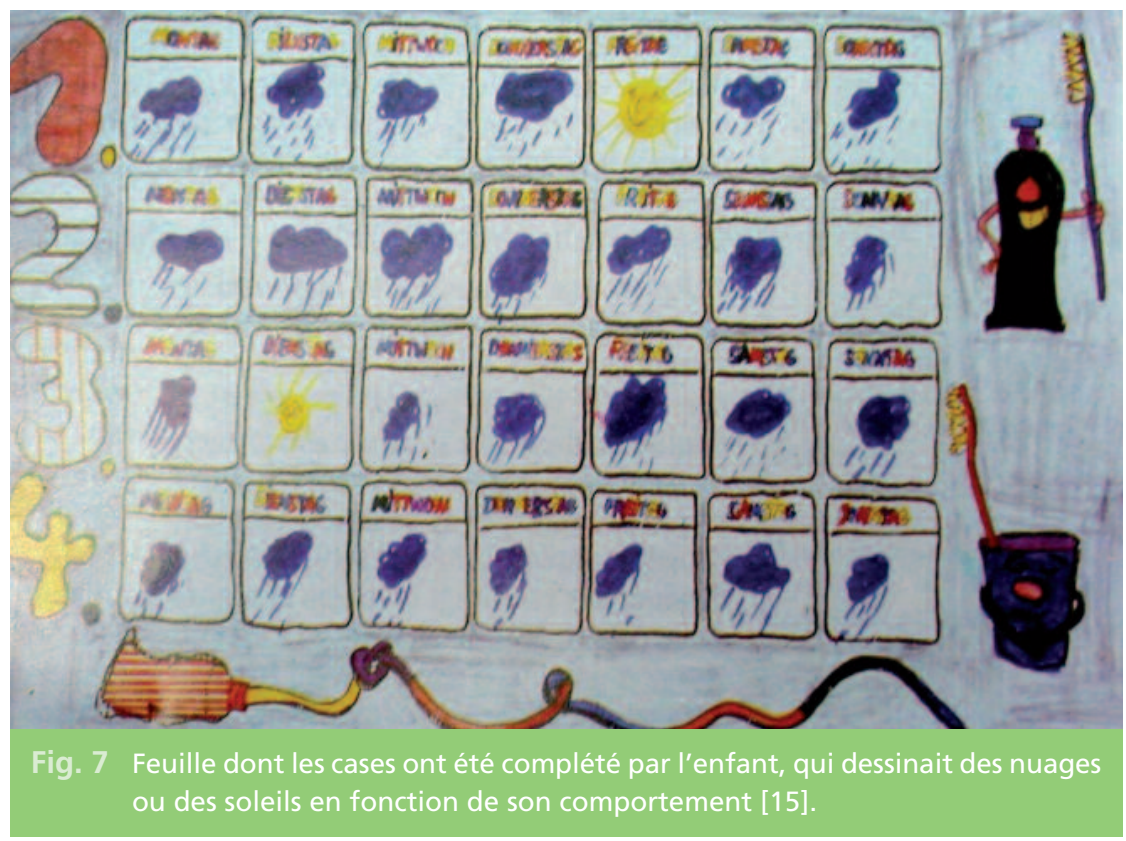



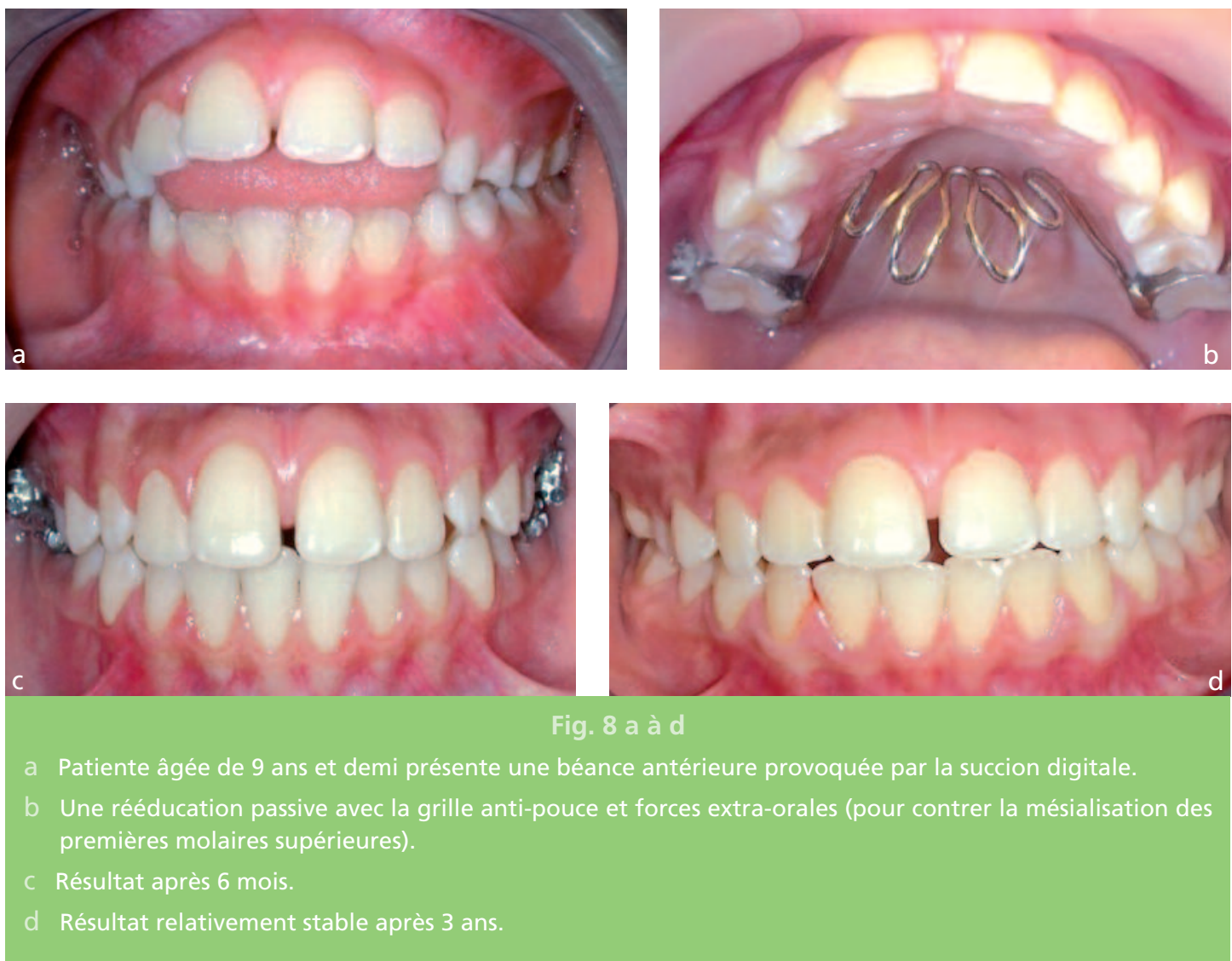

soutenir la coopération de l'enfant, permet également l'arrêt de cette habitude nocive. II s'agit d'une fiche d'autocontrôle assorti d'un système de récompense par jour, semaine et mois, l'enfant met une marque en vert s'il s'est abstenu de sucer son pouce $[13,14]$.

Certaines déformations maxillaires sont transitoires et disparaissent dans les semaines qui suivent l'arrêt de la succion [15]. Certains spécialistes estiment que si l'enfant suce toujours

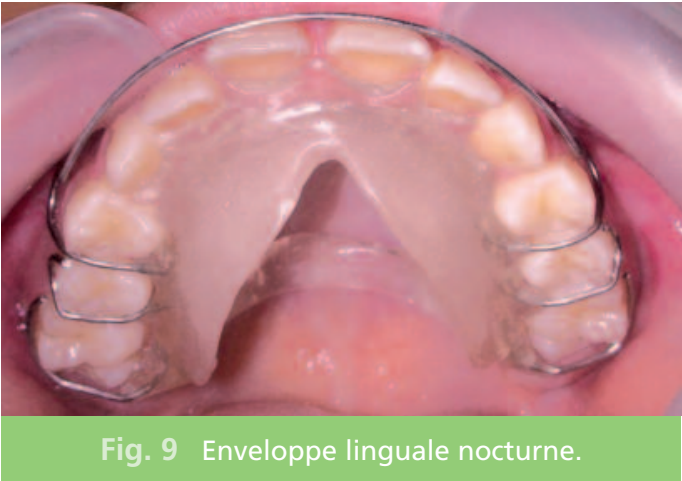
son pouce après 5 ans, un traitement orthodontique multi-attache pourra être nécessaire. Mais celui-ci ne pourra être entrepris que quand l'enfant aura définitivement arrêté de sucer son pouce.

L'étude de M. Velila Heimer [15] a montré la correction de la béance antérieure après l'arrêt de I'habitude de succion [15].

Si malgré tout l'habitude de la succion se prolonge, I'orthodontiste peut placer un appareil amovible tel que : la grille anti-pouce (fig. 8) soudée sur le transpalatin ou l'arc lingual ou encore l'enveloppe linguale nocturne (fig. 9) qui a pour effet de rééduquer de manière passive la position de la langue [16]. 
Le succès de la thérapeutique orthodontique ne prouve pas que le problème étiologique, psychologique et affectif, soit résolu dans toute sa complexité, il faut donc un accompa- gnement psychologique de l'enfant pour l'arrêt de la succion qui doit être antérieur au traitement orthodontique.

\section{Conséquences de la succion prolongée}

Le besoin de succion est fondamental. Outre sa fonction dans l'alimentation pendant les six premiers mois de la vie, le fait de sucer permet à l'enfant de se calmer, de maîtriser ses émotions et de trouver le sommeil.

L'arrêt de cette habitude nocive ne peut être obtenu si l'enfant ne reconnaît pas ses effets néfastes. La participation des parents et de l'entourage aide grandement l'enfant à se débar- rasser de cette habitude, il faut donc l'encourager, voire même le récompenser.

L'orthodontiste peut encourager l'enfant dans ce sens, il peut également faire prendre conscience à l'enfant des conséquences réelles de la succion sur ses dents. Si toutefois cette approche n'est pas efficace, il peut recommander un appareil dentaire empêchant la succion.

\section{Bibliographie}

1. Lupi-Pegurier $\mathrm{L}$, Muller-Bolla M.

Facteurs de risque

et conséquences

bucco-dentaires

de la succion des doigts :

enquête épidémiologique. Int Orthod 2004,23:75-87.

2. Couly G.

La succion,

indice qualitatif

de maturation néonatale.

Arch Fr Pediatr

1985;42:743-5.

3. Foray H, Le Borgne G.

De la succion

à la mastication,

une question

de stimulation.

J Odonto Stomatol Pédiatr 1999;9(1):43-50.
4. Fellus $P$.

Orthodontie précoce en denture temporaire.

Paris : CdP, 2003.

5. Fellus P, Helewa A.

La succion du pouce.

Clinic 2006;27:197-200.

6. For the dental patient...

Thumb sucking

and pacifier use.

J Am Dent Assoc

2007;138:1176.

7. Nowak AJ.

Feeding and

dentofacial development.

J Dent Res

1991,70:159-60.

8. Ngom PI, Diagne F, Samba Diouf J, Ndiaye A, Hennequin $\mathrm{M}$.
Prévalence et facteurs

associés aux habitudes

de succion non nutritive.

Étude transversale

chez des enfants sénégalais

âgés de 5/6 ans.

Orthod Fr 2008;79:99-106.

9. Berthet $A$, François $C$,

Barthelemi S.

Les dysfonctions

oro-faciales :

dépister et intercepter

entre 0 et 7 ans.

J Odonto-Stomatol Pédiatr 1999;9(1):51-66.

10. Courson F.

Orthopédie dento-faciale chez le jeune enfant.

Odontologie pédiatrique /

Archives de pédiatrie

2006;13:678-84. 
11. Singh $S P$, Utreja $A$,

Chawla HS.

Distribution of

malocclusion types

among thumb suckers

seeking orthodontic

treatment.

$\mathrm{J}$ Indian Soc Pedod Prevent

Dent 2008;26:S114-S117.

12. Yokota R, Mishiro M, Abe T, Miyake A, Shiina N, Sueishi $\mathrm{K}$, Yamaguchi $\mathrm{H}$.

Pressure on anterior region of palate during thumb-sucking. Bull Tokyo Dent Coll 2007;48(2):57-66.
13. Djaha K, Assi KD, Bamba M, Toure SH.

Thérapeutiques interceptives des comportements oraux néfastes de l'enfant.

Odontostomatol Trop 1996;19(75):22-3.

14. Günter Sander F., Weinreich $A$.

Une nouvelle méthode pour arrêter la succion du pouce. Rev Orthop Dento Faciale 1989;23(2):227-34.

15. Heimer MV,
Tornisiello Katz CR, Rosenblatt A.

Non-nutritive sucking habits, dental malocclusion, facial morphology in brazilian children: a longitudinal study. Eur J Orthod 2008;30:580-5.

16. Todorova I. Orthopédie préventive et interceptive.

Paris : Elsevier.

Encycl Méd Chir,

(Elsevier, Paris),

Odontologie/Stomatologie, 1999, 23-405-E-10, 8 p.

\section{SUMMARY}

\section{Non nutritive sucking habits}

Afaf HOUB-DINE, Loubna BAHIJE, Mustapha EL ALLOUSSI, Fatima ZAOUI

\section{Keywords \\ - sucking habit \\ thumb sucking \\ - pacifier sucking}

Sucking behaviours have been described in the literature under two facets: nutritive and non nutritive. Nutritive sucking refers to breast feeding, bottle feeding or a combination of both. Non nutritive sucking habits are mentioned when children suck their thumb or another finger(s), a pacifier or any other object.

Non nutritive sucking habits are very frequent in the young child. Although digital sucking in less than five-year-old child should not be alarming, it may generates variable degrees of malocclusions as the permanent teeth make their eruption.

Thus, non nutritive sucking appears complex, in its consequences as well as in its global treatment. Several processes and devices used to stop this deforming habit have been inducing many discussions over literature. The educational and psychological methods remain up to date. 